\title{
Size-Dependent Disorder-Order Transformation in the Synthesis of Monodisperse Intermetallic PdCu Nanocatalysts
}

Chenyu Wang, ${ }^{1}$ Dennis P. Chen, ${ }^{1}$ Xiahan Sang, ${ }^{2}$ Raymond R. Unocic, ${ }^{2}$ and Sara E. Skrabalak ${ }^{1, *}$

${ }^{1}$ Department of Chemistry, Indiana University, 800 East Kirkwood Avenue, Bloomington, Indiana 47405, United States

${ }^{2}$ Center for Nanophase Materials Sciences, Oak Ridge National Laboratory, One Bethel Valley Road, Oak Ridge, TN 37831 USA

\section{$\underline{\text { Table of Contents }}$}

STEM micrographs/EDS-mapping of PdCu A1 NPs (Figure S1)

Elemental analysis of PdCu A1 NPs obtained from the STEM-EDX spectrum (Table S1) S3

TEM images and XRD patterns of annealed PdCu A1 NPs (Figure S2)

XPS spectra of Pd 3d for PdCu B2 NPs before and after ion sputtering (Figure S3)

TEM image and XRD patterns of PdCu NPS synthesized by seed-mediated co-reduction S4 with $\mathrm{Pd}(\mathrm{acac})_{2}$ as precursors (Figure S4)

Profile-fitting of XRD patterns obtained from quenched seed-mediated co-reduction S5 (Figure S5)

Relative intensity of the A1 (111) and B2 (110) diffraction peaks as a function of time S5 (Figure S6)

Powder XRD pattern TEM image of PdCu A1 NPs aged in OAm-TOP solution (Figure S6 S7)

TEM image of $5 \mathrm{~nm}$ Pd NPs; (B) Powder XRD pattern of $5 \mathrm{~nm}$ Pd NPs (Figure S8) 
ORR polarization curves of PdCu B2 NPs and PdCu A1 NPs before and after 3000 S7 potential cycles (Figure S9)

TEM images of PdCu A1 and B2 NPs before and after 3000 potential cycles (Figure S7 S10)
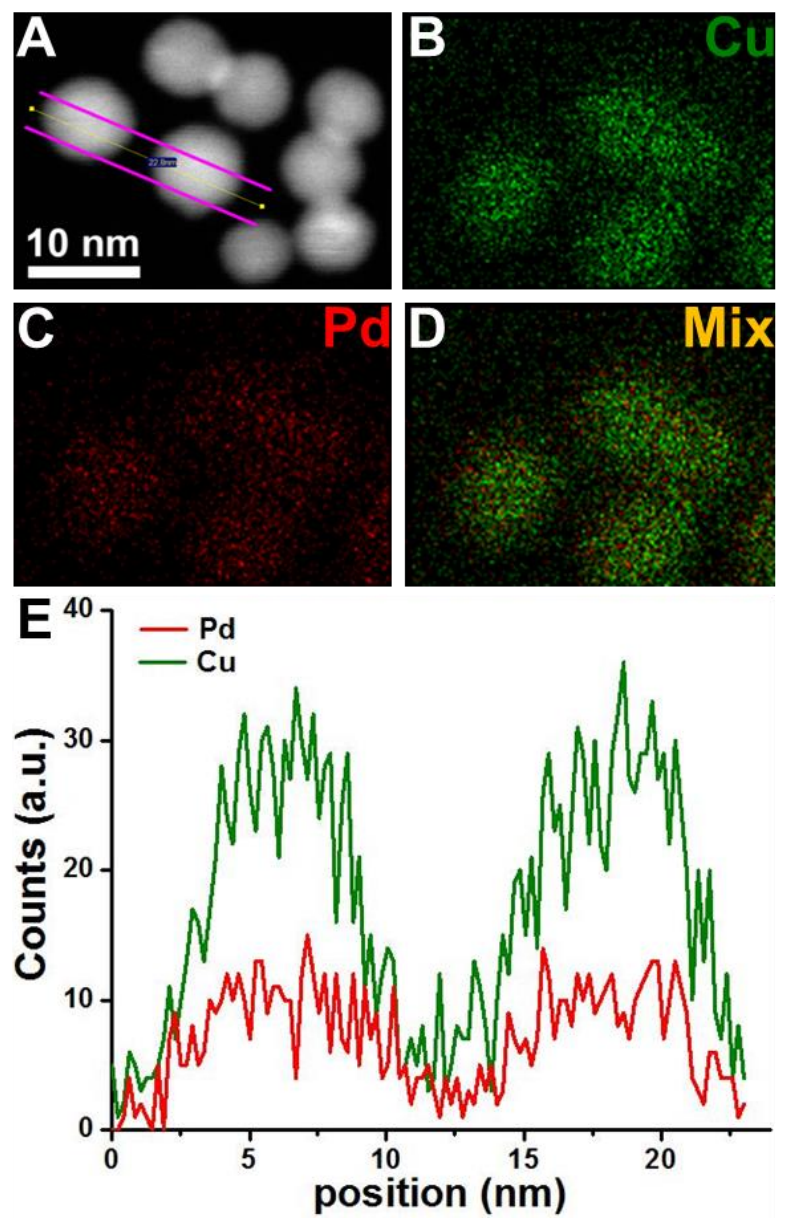

Figure S1. (A) STEM image of selected PdCu A1 NPs for STEM-EDX analysis. Elemental mapping of selected area denotes the presence of (B) $\mathrm{Cu}$ (green), (C) $\mathrm{Pd}$ (red), (D) combined $\mathrm{Cu}$ and $\mathrm{Pd}$. In (E), an EDX line scan corresponding to the yellow line across two particles in (D). 
Table S1. Elemental analysis of PdCu A1 NPs obtained from the STEM-EDX spectrum

\begin{tabular}{|c|c|c|}
\hline Element & Weight \% & Atomic \% \\
\hline $\mathrm{Pd}$ & 36.32 & 49.21 \\
\hline $\mathrm{Cu}$ & 63.68 & 50.79 \\
\hline Total & 100 & 100 \\
\hline
\end{tabular}
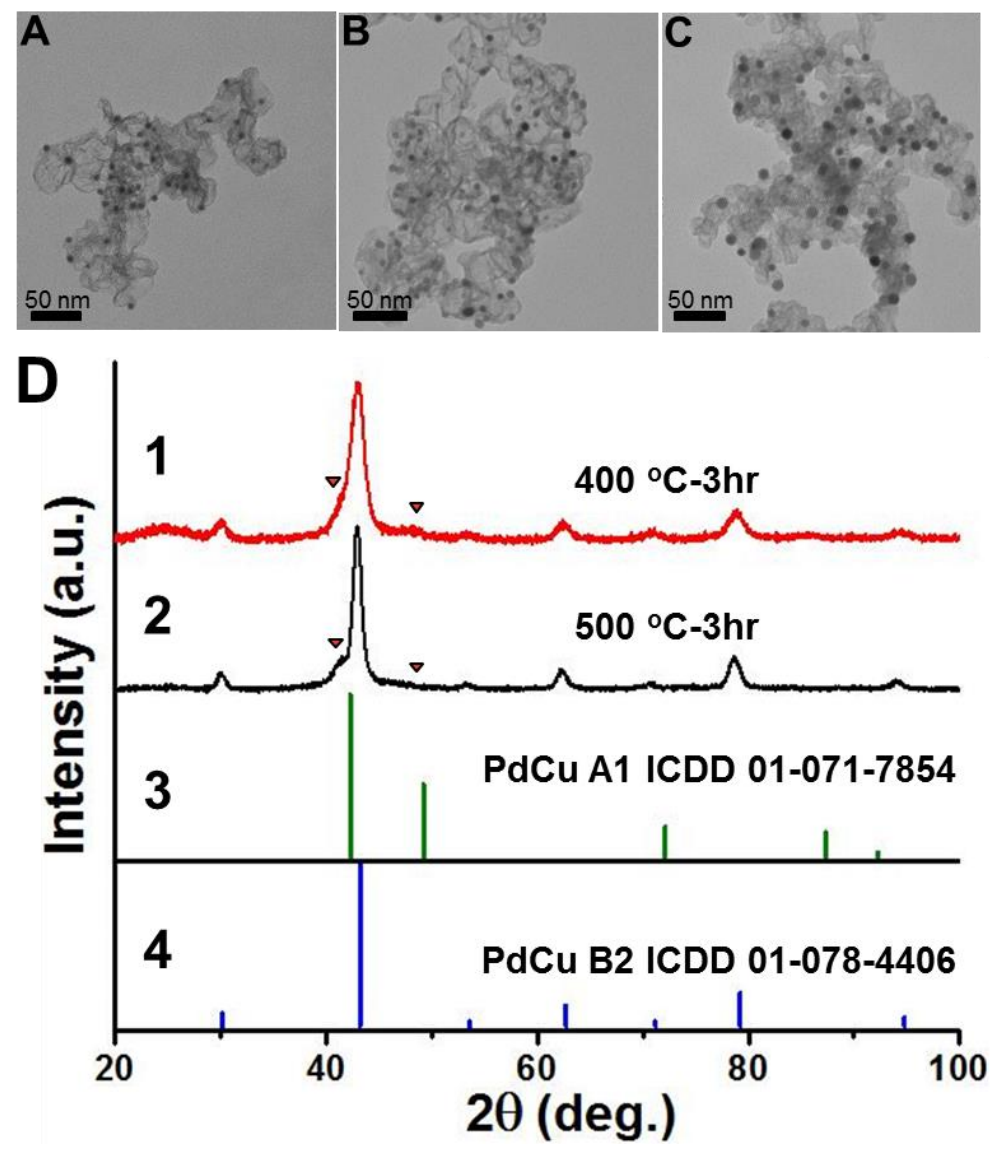

Figure S2. (A) is the TEM image of PdCu A1 NPs loaded on activated carbon before annealing; (B) and (C) are TEM images of these NPs after being annealed for 3 hours at $400{ }^{\circ} \mathrm{C}$ and $500{ }^{\circ} \mathrm{C}$, respectively; (D) 1) and 2) are XRD patterns of annealed PdCu NPs as shown in (A) and (B), respectively; 3) and 4) are reference PdCu A1 (ICDD 01-0717854) and B2 (ICDD 01-078-4406) patterns, respectively. 


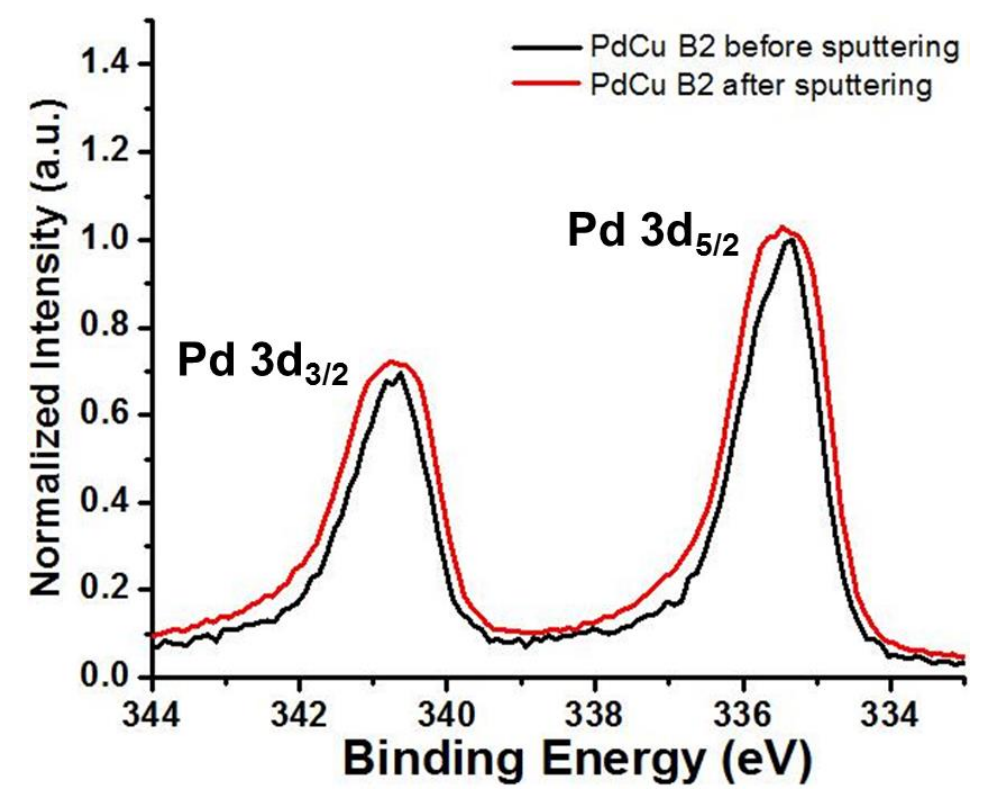

Figure S3. XPS spectra of Pd 3d for PdCu B2 NPs before and after ion sputtering.
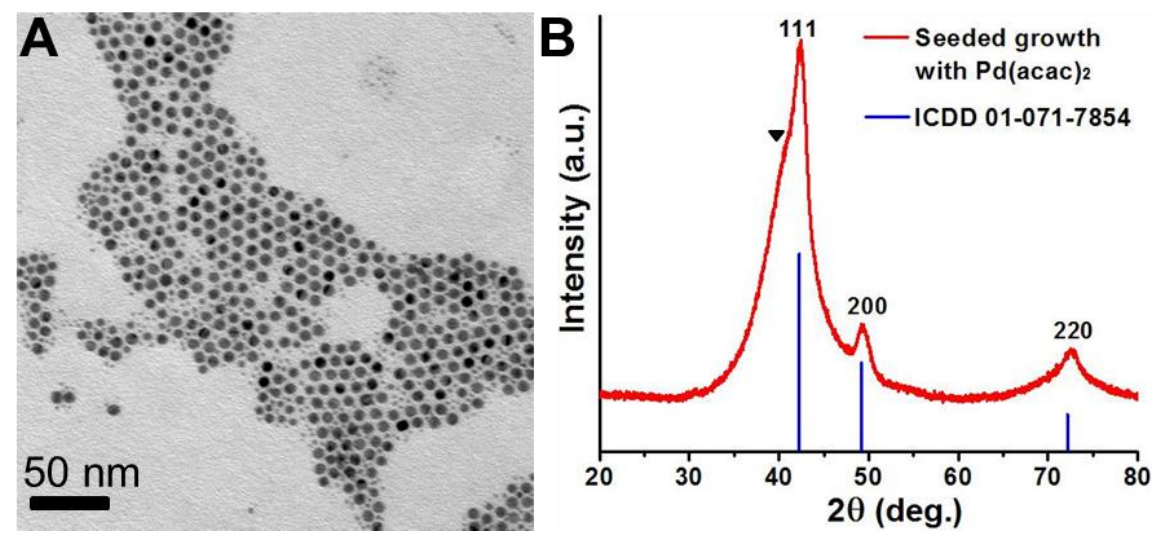

Figure S4. (A) TEM image of $\mathrm{PdCu}$ NPs synthesized through seed-mediated coreduction by using $\mathrm{Pd}(\mathrm{acac})_{2}$ as precursors; (B) Powder XRD pattern of the PdCu NPs shown in (A). 

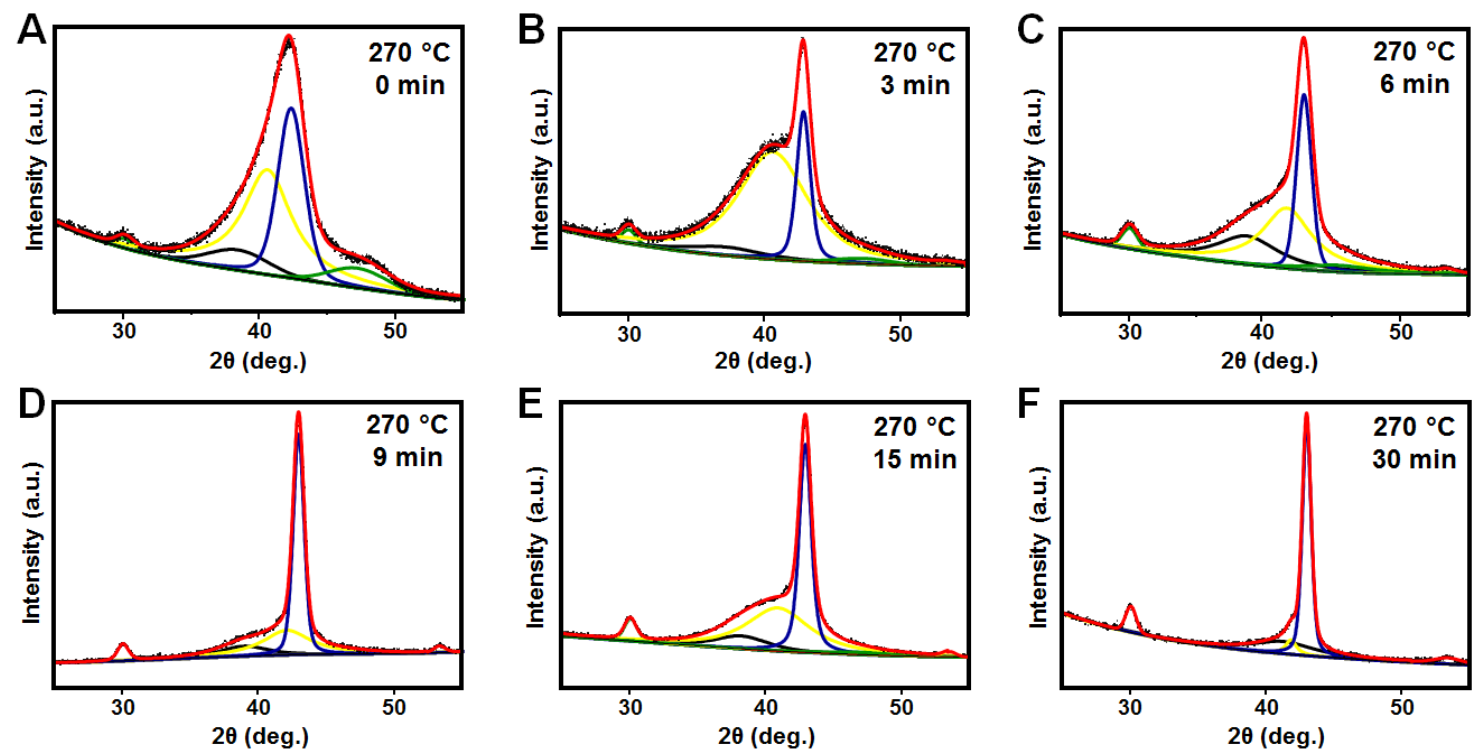

Figure S5. Profile-fitting of XRD patterns obtained from quenched seed-mediated coreduction. The principal peak is deconvoluted into $\mathrm{Pd}$ (111), $\mathrm{PdCu} \mathrm{A} 1$ (111) and $\mathrm{PdCu}$ B2 (110), represented as black, yellow, and blue lines. The calculated pattern is overlaid on the experimental pattern as a solid red line. Additional peaks around $30^{\circ}, 48^{\circ}, 54^{\circ} 2 \theta$ correspond to B2 (100), A1 (200) and B2 (111) reflections, respectively.

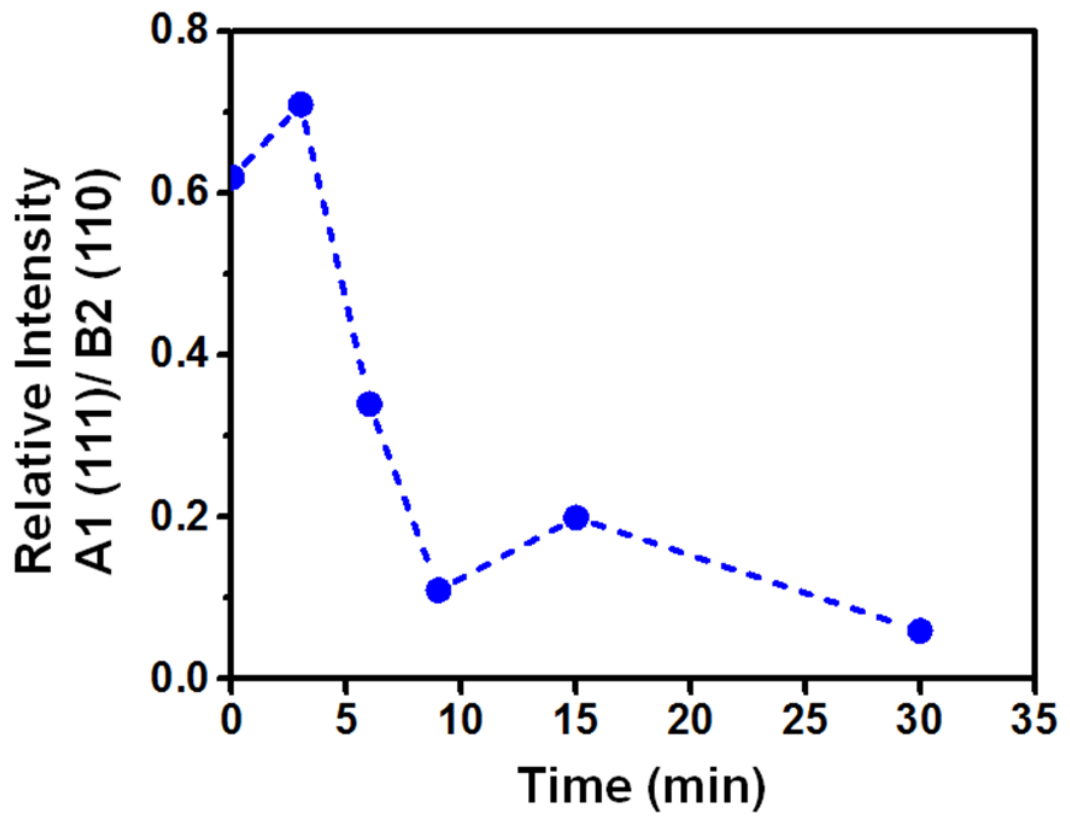

Figure S6. Relative intensity of the A1 (111) and B2 (110) diffraction peaks as a function of time. 

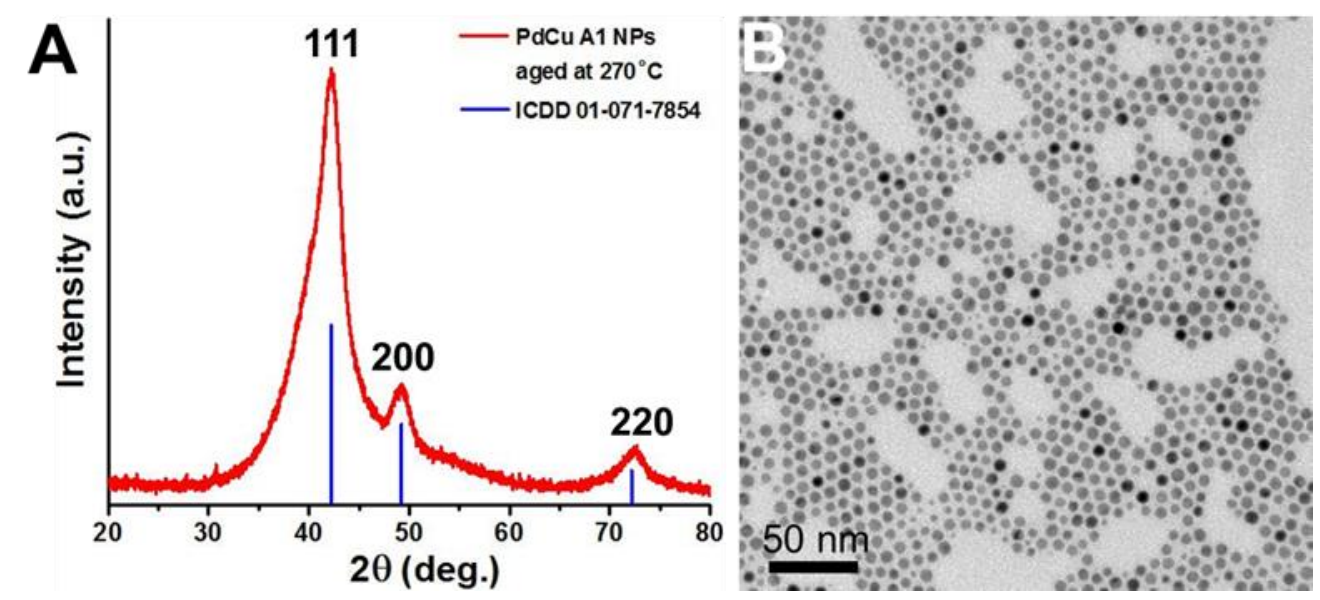

Figure S7. Powder XRD pattern (A) and TEM image (B) of PdCu A1 NPs aged in OAmTOP solution at $270{ }^{\circ} \mathrm{C}$ for 1 hour.
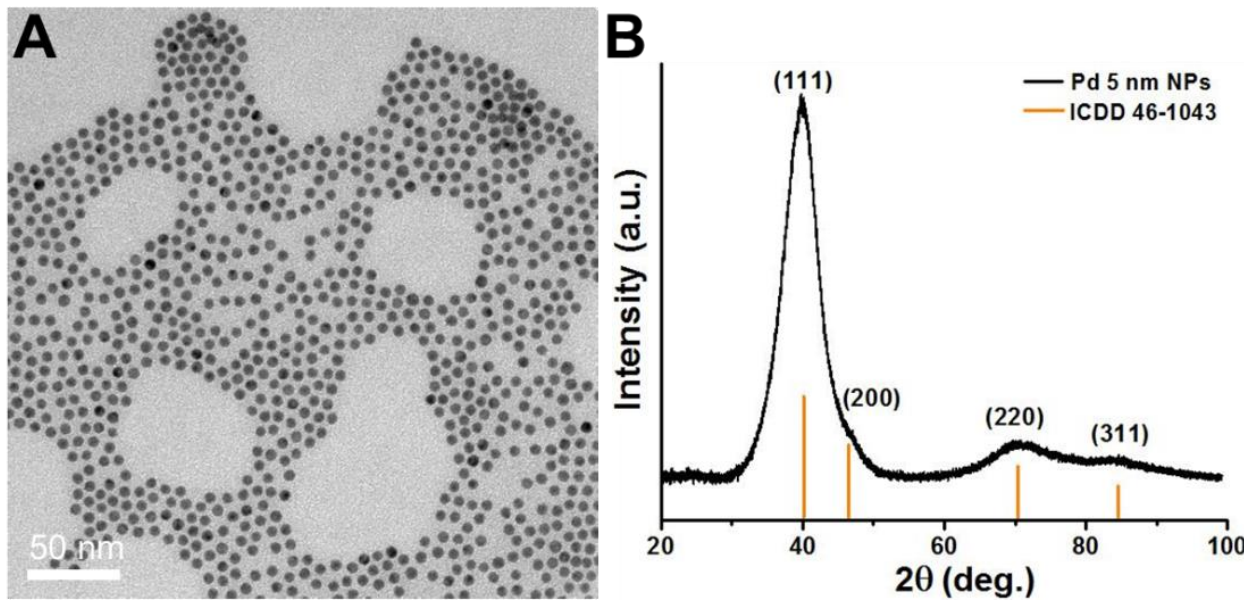

Figure S8. (A) TEM image of $5 \mathrm{~nm}$ Pd NPs; (B) Powder XRD pattern of $5 \mathrm{~nm}$ Pd NPs. 


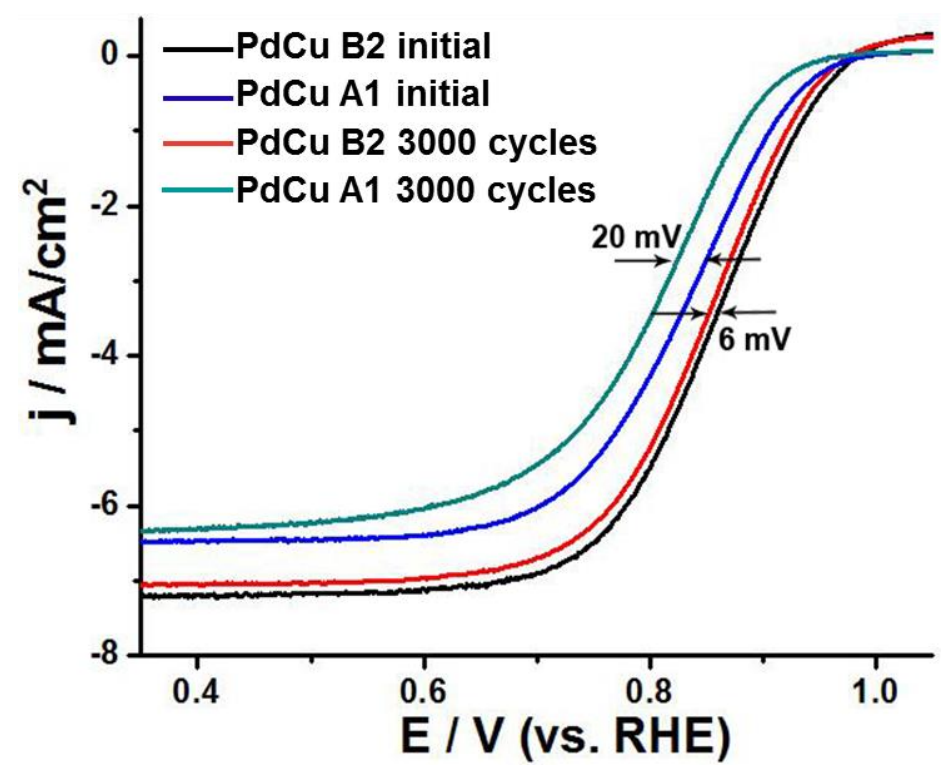

Figure S9. Comparative ORR polarization curves of PdCu B2 NPs and PdCu A1 NPs before and after 3000 potential cycles.
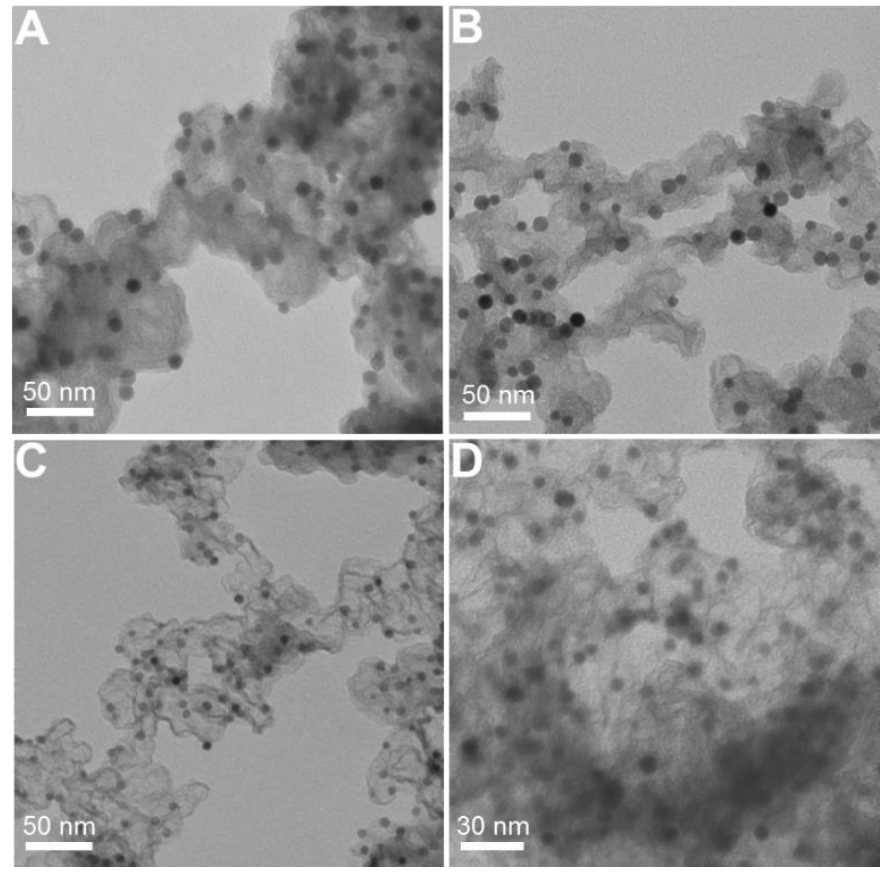

Figure S10. TEM images of PdCu B2 NPs loaded on activated carbon before (A) and after (B) durability test; PdCu A1 NPs loaded on activated carbon before (A) and after (B) durability test. 